\title{
SURFACE WAVE INCIDENCE ON A PLANE STRUCTURE HAVING A MULTI-MODE DISCONTINUITY IN IMPEDANCE*
}

\author{
BY \\ RICHARD C. MORGAN (St. John's University) \\ AND \\ SAMUEL N. KARP (New York University)
}

\begin{abstract}
The phenomenological theory of multi-mode surface wave propagation is applied to a plane structure having a multi-mode discontinuity in impedance. The resulting boundary-value problem is reduced to the solution of a Wiener-Hopf equation whose factorization is given in terms of the factorization that occurred in the one-mode case. Despite the complexity of the solution, the magnitudes of the surface wave excitation coefficients are elementary functions, as is the cylindrical power flow.

On computing the power flow through the impedance surface, a definition of subsurface power flow "inside" the structure is suggested. The form can be taken so that the concept of modal power flow separability is maintained wherever the associated exterior field is primarily that of surface waves. It is further observed that without consideration of this term, power flow coupling occurs. Analogous results appear in the exact case of a dielectric slab having multiple simultaneously propagating surface wave modes.

Lastly, conservation of power is verified by actual evaluation of the closed contour integral used to define the various components of power flow (surface wave, cylindrical, junction, and boundary). In fact, the following physical interpretation can be made for the magnitudes of the respective power flow distributions: the incident surface wave power (including the associated subsurface power) is equal to the excited surface wave power (including the associated subsurface power) plus the cylindrically radiating power.

Introduction. Most exact problems of electromagnetic wave propagation are intractable. As a result, numerous approximate methods have been devised to discuss various aspects of the phenomena. In certain problems involving surface waves, it is known that this feature may be investigated by replacing the details of the structure with an impedance boundary condition.

The class of problems to which we direct ourselves is characterized by discontinuous plane structures (infinite in extent) having the capability of supporting several surface wave modes. The orientation and geometry are such that the electromagnetic field produced is determined by solving a two-dimensional problem and all field components are derivable from a single unknown scalar wave function $u(x, y)$.

The plane structure will be replaced by the boundary conditions put forth by Karp

* Received September 18, 1971. The research reported in this paper was supported partially by St. John's University and partially by the office of Naval Research under Contract No. N00014-67A$0467-0075$. Reproduction in whole or part permitted for any use of the U. S. Government.
\end{abstract}


and Karal [1], [2]. These conditions have the form of generalizing the classical impedance condition to products of this type. Furthermore, they are derivable by approximating the reflection coefficient due to a plane incident wave (which may be known theoretically or experimentally). Previously, these conditions were applied to continuous structures in the papers [2], [3] and [4]. A discontinuity in the boundary condition for a two-mode problem on a right-angled wedge was treated in [5]. This paper will be directed toward solving the resulting mathematical problem of a two-mode discontinuity on a plane structure. A similar single-mode discontinuity problem was discussed by Kay [6]. It is interesting that despite the complexity of the solution, the physically important magnitude of the surface wave excitation coefficients is easily obtained (Kay observed this in the single-mode case). We take as our incident field an appropriate surface wave, and for simplicity, one half of the structure is allowed to be slightly thick (see [4]) while the other half is perfectly conducting.

The various components of the power flow above the structure are computable by choosing an appropriate contour in physical space. In particular, on computing the power flow through the impedance surface, we are led to a definition of power flow inside and down the structure. The form is such that the concept of modal power flow separability is maintained wherever the associated exterior field is primarily that of surface waves. It is well known that, in the exactly solvable case of an infinite dielectric slab having simultaneously several propagating surface wave modes, surface wave modal power flow separability results if the power flow across a plane perpendicular to the slab is computed by including the associated power flow inside. Otherwise, there is in general a coupling of power when it is computed only above the slab. This phenomenon is observed also in our impedance model. For the case of the single real impedance, a consideration of this type does not occur because power is not transferred across the impedance surface and the model is one for which the power flowing inside the structure is negligible. In the case of the dielectric slab mentioned above, this corresponds to a slab of vanishing thickness. However, for a slab of finite thickness, the power flow inside may be significant. Thus, by our model, we provide an approximation that accounts for such power flow while the structure is also discontinuous. The exact problem of such a discontinuous structure has not been solved. However, we point out that the phenomenological theory is broader in context than the problem of the dielectric slab. Thus, we conjecture that our results herein are also.

As a partial verification of our computations, conservation of power is checked for the contour used to define the various component field power distributions (i.e., surface wave, cylindrical and boundary). It is shown that the surface wave power (including that amount associated with flow inside the boundary) exactly cancels out the cylindrically radiated flow. The remaining boundary terms give zero power flow. As a result, we can state the following physical theorem for the magnitudes of the power flow distributions: the incident surface wave power flow (including "inside" term) is equal to the excited surface wave power flow (including "inside" term) plus the cylindrically radiated power flow.

The boundary-value problem formulation is modeled after that used previously in proving uniqueness for a multi-mode right-angled wedge problem [11]. In addition, the junction condition assumed arises naturally upon reducing the problem to the solution of a Wiener-Hopf equation. Here the factorization is given in terms of the factorization that occurred in the one-mode case. 
1. B.V.P. formulation and solution. The problem (see Fig. 1) we shall solve is posed by the following conditions:

(i) $\left(\Delta+\kappa^{2}\right) u(x, y)=0$, all $x, y>0$

(ii) $\left(\partial / \partial y+\lambda_{1}\right)\left(\partial / \partial y+\lambda_{2}\right) u=0, \quad x<0, \quad y=0$, where $\lambda_{1}, \lambda_{2}$ are taken as real positive constants.

(iii) $u=0$, $x>0, \quad y=0$.

(iv) $u$ and its derivatives satisfy
(a) $\sum_{i=0}^{3} \sum_{i=0}^{i}\left|\frac{\partial^{i} u}{\partial x^{i-i} \partial y^{i}}\right|<M$
for $r>R_{0}$,

where $M$ is independent of $\mathrm{r}$ and $\theta$ and $R_{0}$ is some positive constant.

(b) $\partial^{2} u / \partial y^{2}$ is integrable at the origin.

(v) $u=u_{\text {incident }}+u_{\text {excited }}+u_{\text {radiated }}$

$$
\begin{array}{rlr}
u_{\mathrm{inc} .} & =A \exp \left[-\lambda_{1} y+i\left(k^{2}+\lambda_{1}^{2}\right)^{1 / 2} x\right], & \\
& =0<0, y \geq 0, \\
& , \quad x>0, y \geq 0,
\end{array}
$$

and

$$
\begin{array}{rlrl}
u_{\text {ex oi ted }} & =\sum_{m=1}^{2} C_{m} \exp \left[-\lambda_{m} y-i\left(k^{2}+\lambda_{m}^{2}\right)^{1 / 2} x\right], & & x<0, y \geq 0, \\
& =0 \quad, \quad x>0, y \geq 0 .
\end{array}
$$

Here $A$ represents the given incident surface wave amplitude and the $C_{m}$ are constants that must be determined.

(vi) $u_{\text {rndinted }} \equiv u-u_{\text {ino. }}-u_{\text {exoi ted }}$ obeys the radiation condition

$$
\lim _{r \rightarrow \infty} \sqrt{ } r\left(\partial u_{\mathrm{rad} .} / \partial r-i k u_{\mathrm{rad} .}\right)=0
$$

uniformly in $\theta, 0 \leq \theta \leq \pi$.

Following a method employed by Kane and Karp [7] and Kane [8], the solution is reduced to solving a Wiener-Hopf problem. The critical factorization required is that of the function

$$
\sigma\left(\nu ; \lambda_{1}, \lambda_{2}\right)=\prod_{m=1}^{2}\left[1+\frac{\lambda_{m}}{i\left(k^{2}-\nu^{2}\right)^{1 / 2}}\right]
$$

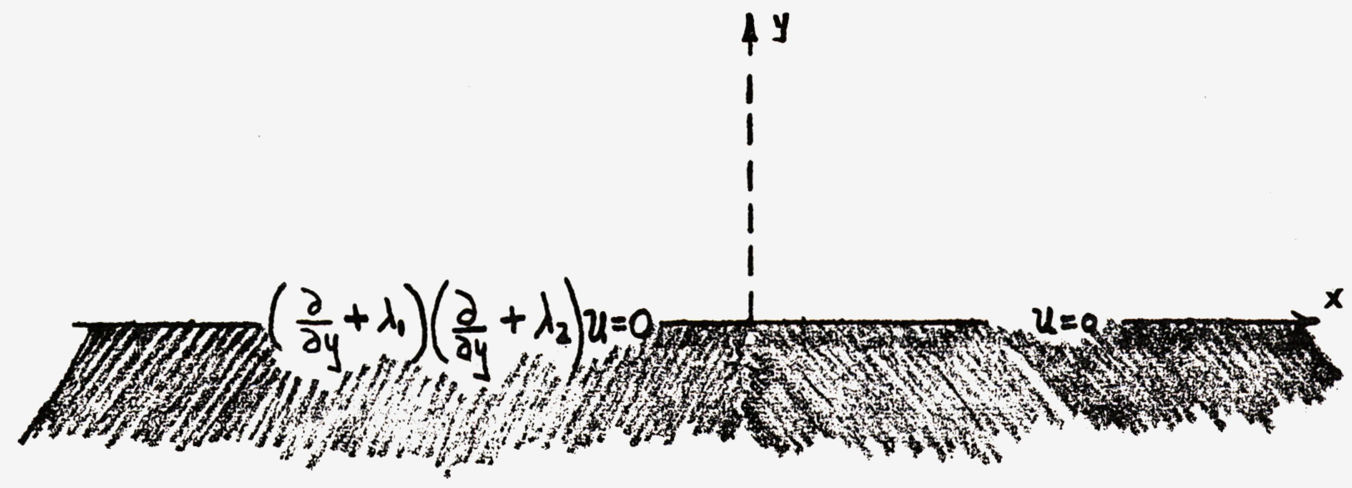

FIG. 1. 
into the quotient

$$
\sigma^{+}\left(\nu ; \lambda_{1}, \lambda_{2}\right) / \sigma^{-}\left(\nu ; \lambda_{1}, \lambda_{2}\right)
$$

where $\sigma^{+}\left(\nu ; \lambda_{1}, \lambda_{2}\right)$ and $\sigma^{-}\left(\nu ; \lambda_{1}, \lambda_{2}\right)$ are respectively analytic in the region $\operatorname{Im} \nu \geq-$ $\operatorname{Im} k$ and $\operatorname{Im} \nu \leq+\operatorname{Im} k$. In principle, the factorization can be done. However, the resulting expressions are exceedingly complicated. Fortunately, the factorization of

$$
\sigma(\nu ; \lambda)=\left[1+\frac{\lambda}{i\left(k^{2}-\nu^{2}\right)^{1 / 2}}\right]
$$

has been given by a number of writers including Fock [9], Bazer and Karp [10] and Kay [6]. Thus, the required factorization may be written in terms of products of the above factors.

The solution is given in the following form:

$$
\begin{aligned}
& u(x, y)=A \exp \left[-\lambda_{1} y+i\left(k^{2}+\lambda_{1}^{2}\right)^{1 / 2} x\right] \\
& +\frac{i A\left(\left(k^{2}+\lambda_{1}^{2}\right)^{1 / 2}+k\right)}{2 \pi} \sigma^{+}\left(\left(k^{2}+\lambda_{1}^{2}\right)^{1 / 2}, \lambda_{1}\right) \sigma^{+}\left(\left(k^{2}+\lambda_{1}^{2}\right)^{1 / 2}, \lambda_{2}\right) \\
& \cdot \int_{-\infty}^{+\infty} \frac{\exp \left[i v x+i\left(k^{2}-\nu^{2}\right)^{1 / 2} y\right]}{\left(\nu-\left(k-\lambda_{1}^{2}\right)^{1 / 2}\right)^{2}}
\end{aligned}
$$

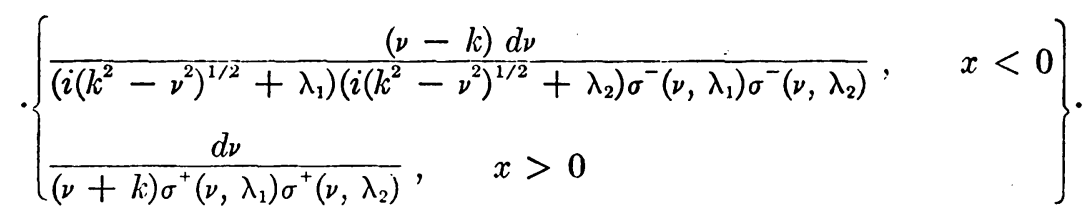

The decomposition for $x>0$ and $x<0$ is strictly for convenience, while the functions $\sigma^{+}(\nu, \lambda)$ and $\sigma^{-}(\nu, \lambda)$ are the factors of (1.3), i.e.

$$
\sigma(\nu, \lambda)=\sigma^{+}(\nu, \lambda) / \sigma^{-}(\nu, \lambda),
$$

where $\sigma^{+}(\nu, \lambda)$ is analytic in the upper $\nu$-plane $\operatorname{Im} \nu \geq-\operatorname{Im} k$ and $\sigma^{-}(\nu, \lambda)$ is analytic for $\operatorname{Im} \nu \leq \operatorname{Im} k$. Furthermore, in their respective domains of analyticity, they have been shown to be zeroless and bounded above and below by positive constants for $|\nu| \rightarrow \infty$. We follow the procedure of [10] with regard to the branch cut necessary in the definition of $\left(k^{2}-\nu^{2}\right)^{1 / 2}$ to ensure convergence of the integral. liurthermore, we observe that (1.4) may be differentiated freely under the integral providing $y>0$. To guarantee convergence in computing $\left.\left(\partial^{2} u / \partial y^{2}\right)\right|_{y=0}$, we deform the contour at $\pm \infty$ slightly so that the term exp $i \nu x$ is exponentially damped. This will cause slight variations in the solution representation for $x>0$ and $x<0$ in the case of $\partial^{2} u / \partial y^{2}$.

The actual solution technique employed the mathematical convenience of $\operatorname{Im} k>0$. Consequently, we must now pass to the limit of $\operatorname{Im} k=0$. We observe that in this limit $u$ is continuous at the origin and that $u_{x}$ and $u_{y}$ are bounded. This latter fact follows after obtaining asymptotic expansions for the Weiner-Hopf factors appearing in the integrand of the solution representation. The forms are easily found using the exact expressions given in Kay's paper.

${ }^{1}$ This is the factorization that appears in the single impedance case (see Kay [6]). 
Finally, we note that the incident field is exactly cancelled out by the residue at $\nu=\left(k^{2}+\lambda_{1}^{2}\right)^{1 / 2}$ when $x$ is positive, in fulfillment of condition (v).

2. Discussion of the solution. In the present section we discuss the solution given in Eq. (1.4). It has certain notable features, namely surface wave terms and a cylindrical far field, which will be discussed separately below.

Surface wave modes $A$. We note that for $x<0$ the poles of the integrand determine the excited surface wave contributions and occur at $\nu=-\left(k^{2}+\lambda_{j}^{2}\right)^{1 / 2}, j=1,2 .^{2}$ Thus, writing the excited modes as

$$
c_{1} \exp \left[-\lambda_{1} y-i\left(k^{2}+\lambda_{1}^{2}\right)^{1 / 2} x\right]+c_{2} \exp \left[-\lambda_{2} y-i\left(k^{2}+\lambda_{2}^{2}\right)^{1 / 2} x\right],
$$

we find that

$$
c_{1}=\frac{A \lambda_{1}\left(k+\left(k^{2}+\lambda_{1}^{2}\right)^{1 / 2}\right)^{2}}{2\left(\lambda_{2}-\lambda_{1}\right)\left(k^{2}+\lambda_{1}^{2}\right)} \prod_{i=1}^{2} \frac{\sigma^{+}\left(\left(k^{2}+\lambda_{1}^{2}\right)^{1 / 2}, \lambda_{j}\right)}{\sigma^{-}\left(-\left(k^{2}+\lambda_{1}^{2}\right)^{1 / 2}, \lambda_{i}\right)}
$$

and

$$
c_{2}=\frac{A \lambda_{2}\left(k+\left(k^{2}+\lambda_{1}^{2}\right)^{1 / 2}\right)\left(k+\left(k^{2}+\lambda_{2}^{2}\right)^{1 / 2}\right)}{\left(\lambda_{1}-\lambda_{2}\right)\left(k^{2}+\lambda_{2}^{2}\right)^{1 / 2}\left(\left(k^{2}+\lambda_{1}^{2}\right)^{1 / 2}+\left(k^{2}+\lambda_{2}^{2}\right)^{1 / 2}\right)} \prod_{i=1}^{2} \frac{\sigma^{+}\left(\left(k^{2}+\lambda_{1}^{2}\right)^{1 / 2}, \lambda_{j}\right)}{\sigma^{-}\left(-\left(k^{2}+\lambda_{2}^{2}\right)^{1 / 2}, \lambda_{j}\right)} .
$$

Then, using the formulas given by Kay $[6]^{3}$, we obtain $\left|c_{1}\right|^{2}$ and $\left|c_{2}\right|^{2}$ in the following elementary forms:

$$
\begin{aligned}
& \left|c_{1}\right|^{2}=\frac{|A|^{2} \lambda_{1}^{2}\left(\left(k^{2}+\lambda_{1}^{2}\right)^{1 / 2}+\left(k^{2}+\lambda_{2}^{2}\right)^{1 / 2}\right)^{2}}{\left(\lambda_{1}-\lambda_{2}\right)^{2}\left(k^{2}+\lambda_{1}^{2}\right)} \\
& \left|c_{2}\right|^{2}=\frac{4|A|^{2} \lambda_{2}^{2}\left(k^{2}+\lambda_{1}^{2}\right)^{1 / 2}}{\left(\lambda_{1}-\lambda_{2}\right)^{2}\left(k^{2}+\lambda_{2}^{2}\right)^{1 / 2}} .
\end{aligned}
$$

Cylindrical far field $B$. In the usual way, we make the assumption that $u_{c y 1} \simeq$ $\left(e^{+i k r} /(k r)^{1 / 2}\right) f(\theta)$ and apply the method of steepest descent to obtain

$$
|f(\theta)|^{2}=\frac{|A|^{2}\left(k^{2}+\lambda_{1}^{2}\right)^{1 / 2}\left(\left(k^{2}+\lambda_{1}^{2}\right)^{1 / 2}+\left(k^{2}+\lambda_{2}^{2}\right)^{1 / 2}\right) k^{2} \sin ^{2} \theta}{\pi\left(k \cos \theta-\left(k^{2}+\lambda_{1}^{2}\right)^{1 / 2}\right)^{2}\left(k \cos \theta+\left(k^{2}+\lambda_{1}^{2}\right)^{1 / 2}\right)\left(k \cos \theta+\left(k^{2}+\lambda_{2}^{2}\right)^{1 / 2}\right)}
$$

uniformly in $\theta, 0 \leq \theta \leq \pi$. We observe that (2.6) vanishes for $\theta=0$ and $\pi$. For the latter angle, this represents an example of the lemma, that the radiated far field vanishes to order $(k r)^{-1 / 2}$ on the impedance surface that supports surface waves (see [12]).

3. Power distributions. Aside from a proportionality factor, the real time-average power flow per unit width in the $z$-direction provided by the various components of the field is identifiable by considering the line integral

$$
-\operatorname{Im} \oint u \frac{\partial u}{\partial n} d s
$$

2 These modes will appear respectively for $\pi \geq \theta>\theta_{c}\left(\lambda_{j}\right), j=1,2$. Here $\theta_{c}\left(\lambda_{j}\right)$ is defined by $\theta_{0}=$ $\operatorname{arc} \cos \left(-k /\left(k^{2}+\lambda_{i}^{2}\right)^{1 / 2}\right)$. A more complete discussion of this property is found in [3].

' In effect, we apply the observation of J. Lurye that by considering the physically more important magnitudes of $c_{1}$ and $c_{2}$ a great simplification results. This was done by Kay to give $\left|\sigma^{+}\left(\left(k^{2}+\lambda_{i}^{2}\right)^{1 / 2}, \lambda_{j}\right)\right|^{2} \equiv 1 /\left|\sigma^{-}\left(-\left(k^{2}+\lambda_{i}^{2}\right)^{1 / 2}, \lambda_{j}\right)\right|^{2}=\left(\left(k^{2}+\lambda_{j}^{2}\right)^{1 / 2}+\left(k^{2}+\lambda_{i}^{2}\right)^{1 / 2}\right) /\left(k+\left(k^{2}+\lambda_{i}^{2}\right)^{1 / 2}\right)$.

- Here the normal is taken as positive into the enclosed region. It then follows that the power flow is positive for flow into this region. 
around an appropriate contour. The contour chosen is depicted in Fig. 2. There we take $\theta(R)$ such that as $R \rightarrow \infty, R \sin \theta(R) \rightarrow \infty$ while $\theta(R) \rightarrow 0$. Also, we assume that $c_{\rho} \rightarrow 0$. Consequently, zero power flow occurs at the junction through this contour because of the boundedness of $u$ and its first derivatives.

Cylindrical power flow A. The cylindrical radiated power is defined to be

$$
+\operatorname{Im}\left\{\lim _{R \rightarrow \infty} \int_{0}^{\pi-\theta(R)} u \frac{\partial u}{\partial R} R d \theta\right\} .
$$

As $u \simeq\left(e^{+i k r} /(k r)^{1 / 2}\right) f(\theta), 0 \leq \theta<\pi$, we have the usual form

$$
-\int_{0}^{\pi}|f(\theta)|^{2} d \theta
$$

Thus it is necessary to evaluate the integral

$$
I=\int_{0}^{\pi} \frac{\sin ^{2} \theta d \theta}{\left(\left(k^{2}+\lambda_{1}^{2}\right)^{1 / 2}+k \cos \theta\right)\left(\left(k^{2}+\lambda_{2}^{2}\right)^{1 / 2}+k \cos \theta\right)\left(k \cos \theta-\left(k^{2}+\lambda_{1}^{2}\right)^{1 / 2}\right)^{2}} .
$$

This can be done in standard fashion by introducing the transformation $z=\exp (i \theta)$ and using the evenness of the integrand about $\theta=\pi$ to give

$$
I=\frac{2 i}{k^{4}} \oint_{|z|=1} \frac{\left(z^{2}-1\right)^{2} z d z}{\prod_{i=1}^{2}\left(z^{2}+2\left(1+\left(\lambda_{j} / k\right)^{2}\right)^{1 / 2} z+1\right)\left(z^{2}-2\left(1+\left(\lambda_{1} / k\right)^{2}\right)^{1 / 2} z+1\right)^{2}} .
$$

We note that there are two simple poles inside the unit circle at

$$
z=-\left(1+\left(\lambda_{1} / k\right)^{2}\right)^{1 / 2}+\lambda_{1} / k
$$

and

$$
z=-\left(1+\left(\lambda_{2} / k\right)^{2}\right)^{1 / 2}+\lambda_{2} / k
$$

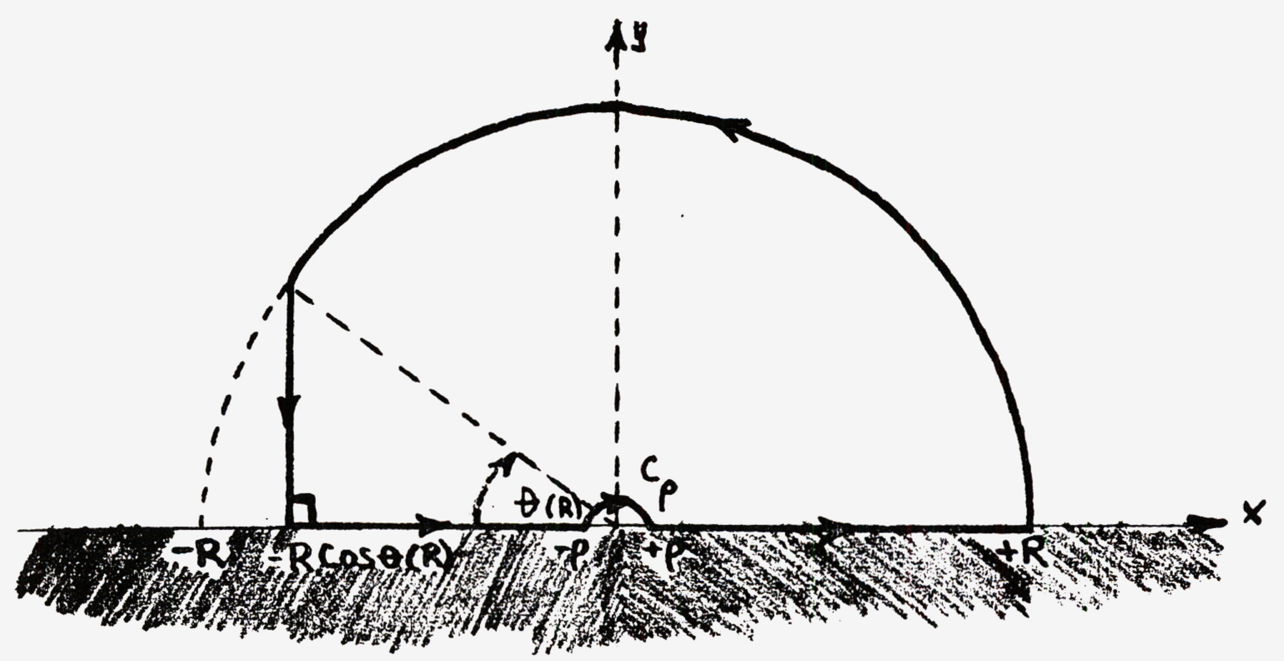

FIG. 2. 
while a double pole occurs at

$$
z=\left(1+\left(\lambda_{1} / k\right)^{2}\right)^{1 / 2}-\lambda_{1} / k
$$

Consequently, on applying residue theory, we obtain

$$
\int_{0}^{\pi}|f(\theta)|^{2} d \theta=\left[\frac{1}{2} \frac{1}{x_{0}\left(1+x_{0}^{2}\right)^{1 / 2}}+\frac{1}{x_{0}\left(1+x_{1}^{2}\right)^{1 / 2}+x_{1}\left(1+x_{0}^{2}\right)^{1 / 2}}\right]|A|^{2},
$$

where $x_{0}=\lambda_{1} / k$ and $x_{1}=\lambda_{2} / k$.

Modal surface wave power flow $B$. The exact case of multi-mode surface wave propagation over a grounded dielectric slab will be used to suggest the form of our definition MSWPF. Here it is known that the power flow across a plane perpendicular to the slab may be computed as the sum of the power flows due separately to each mode, providing that the integrations are carried out above and below the slab (otherwise a coupling effect results) (see Fig. 3). In this regard, it is easily observed that the corresponding phenomenological model of two-mode surface wave propagation over the above exact structure exhibits analogous properties. Since our problem is essentially an exterior one, the question arises as to the definition of the power flow so that analogy with such modal power flow separability results. Physically speaking, what can we use to play the part of the flow inside the slab? Specifically, let us consider such a two-mode problem. Then, for this infinite continuous structure, the solution is given by

$u(x, y)=A_{1} \exp \left[-\lambda_{1} y+i\left(k^{2}+\lambda_{1}^{2}\right)^{1 / 2} x\right]+A_{2} \exp \left[-\lambda_{2} y+i\left(k^{2}+\lambda_{2}^{2}\right)^{1 / 2} x\right]$,

where $A_{1}$ and $A_{2}$ are given. Here no other fields are excited and the incident fields simply propagate down the structure. Now the power across a plane perpendicular to the impedance plane is given by

$$
\begin{aligned}
\operatorname{Im} \int_{\infty}^{0} u \frac{\overline{\partial u}}{\partial x} d y=\left|A_{1}\right|^{2} \frac{\left(k^{2}+\lambda_{1}^{2}\right)^{1 / 2}}{2 \lambda_{1}}+\left|A_{2}\right|^{2} \frac{\left(k^{2}+\lambda_{2}^{2}\right)^{1 / 2}}{2 \lambda_{2}} \\
\quad+\frac{\left(\left(k^{2}+\lambda_{1}^{2}\right)^{1 / 2}+\left(k^{2}+\lambda_{2}^{2}\right)^{1 / 2}\right)}{\left(\lambda_{1}+\lambda_{2}\right)} \operatorname{Re}\left\{A_{1} \bar{A}_{2} \exp +i\left[\left(k^{2}+\lambda_{1}^{2}\right)^{1 / 2}-\left(k^{2}+\lambda_{2}^{2}\right)^{1 / 2}\right] x\right\} .
\end{aligned}
$$

Thus it is easily observed that a coupling of power flow occurs between the modes as, in general, the bracketed term does not vanish. Also, it is easily shown that the integration giving the power flow across any two-mode impedance surface between the arbitrary points $\left(x_{0}, 0\right)$ and $\left(x_{1}, 0\right)$ simplifies to

$$
-\left.\operatorname{Im} \int_{x_{0}}^{x_{1}} u \frac{\partial u}{\partial y}\right|_{y=0} d x=+\operatorname{Im} \frac{1}{\lambda_{1}+\lambda_{2}}\left\{u\left(x_{0}, 0\right) \frac{\overline{\partial u}}{\partial x}\left(x_{0}, 0\right)-u\left(x_{1}, 0\right) \frac{\overline{\partial u}}{\partial x}\left(x_{1}, 0\right)\right\} \text {. }
$$

Thus, if one extends the vertical contours through the impedance surface into a virtual structure at $x=x_{0}$ and $x=x_{1}$ (see Fig. 4), conservation of power suggests that inside the structure the power flow across $x=x_{0}\left(P_{-}\left(x_{0}\right)\right)$ and $x=x_{1}\left(P_{-}\left(x_{1}\right)\right)$ respectively satisfies the following condition:

$$
P_{-}\left(x_{0}\right)=P_{-}\left(x_{1}\right)-\left.\operatorname{Im} \int_{x_{0}}^{x_{1}} \cdot \bar{u} \frac{\partial u}{\partial y}\right|_{y=0} d x .
$$

(This equation also holds in the case of a slab.) Substituting (3.12) into (3.13), we have 

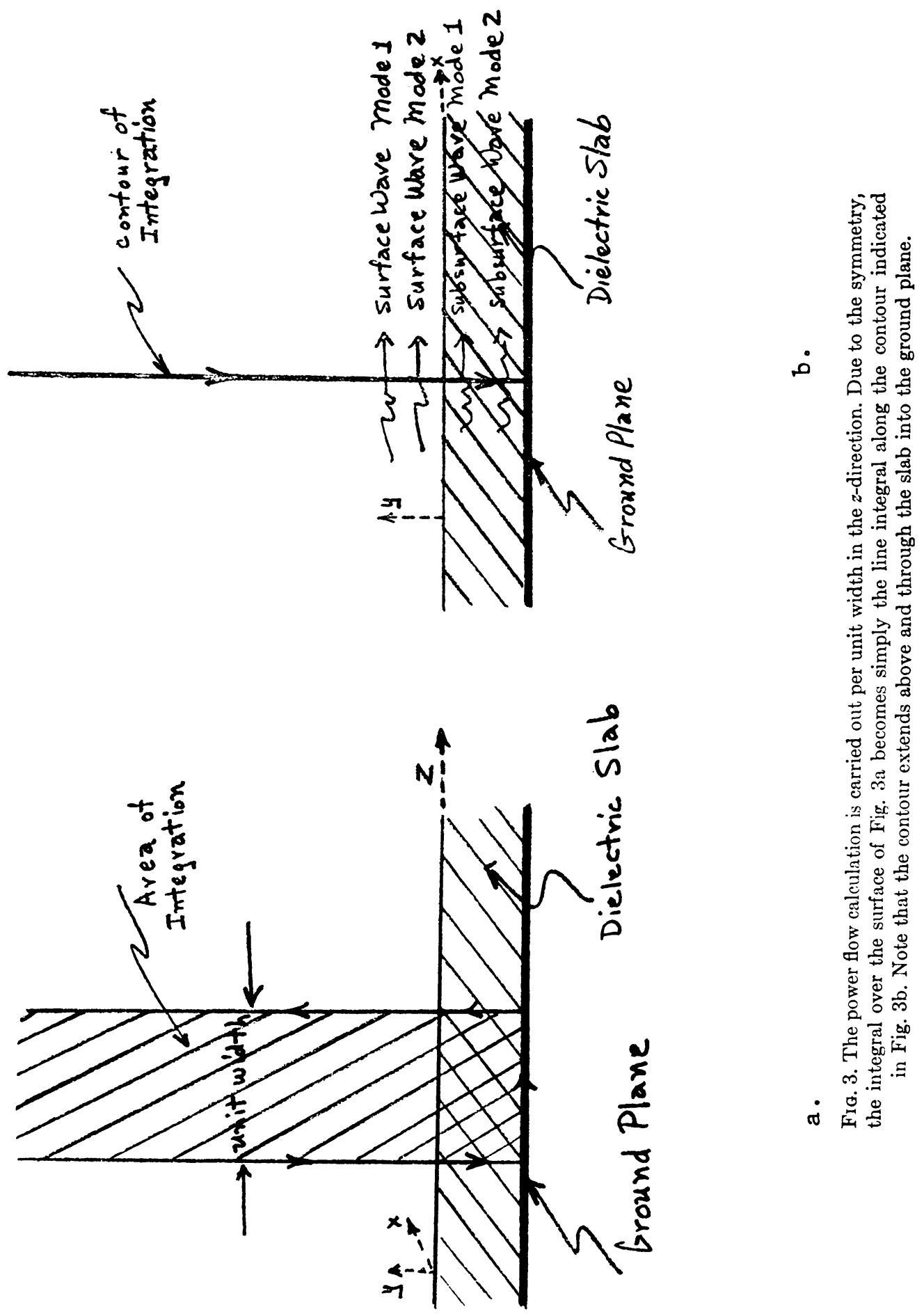


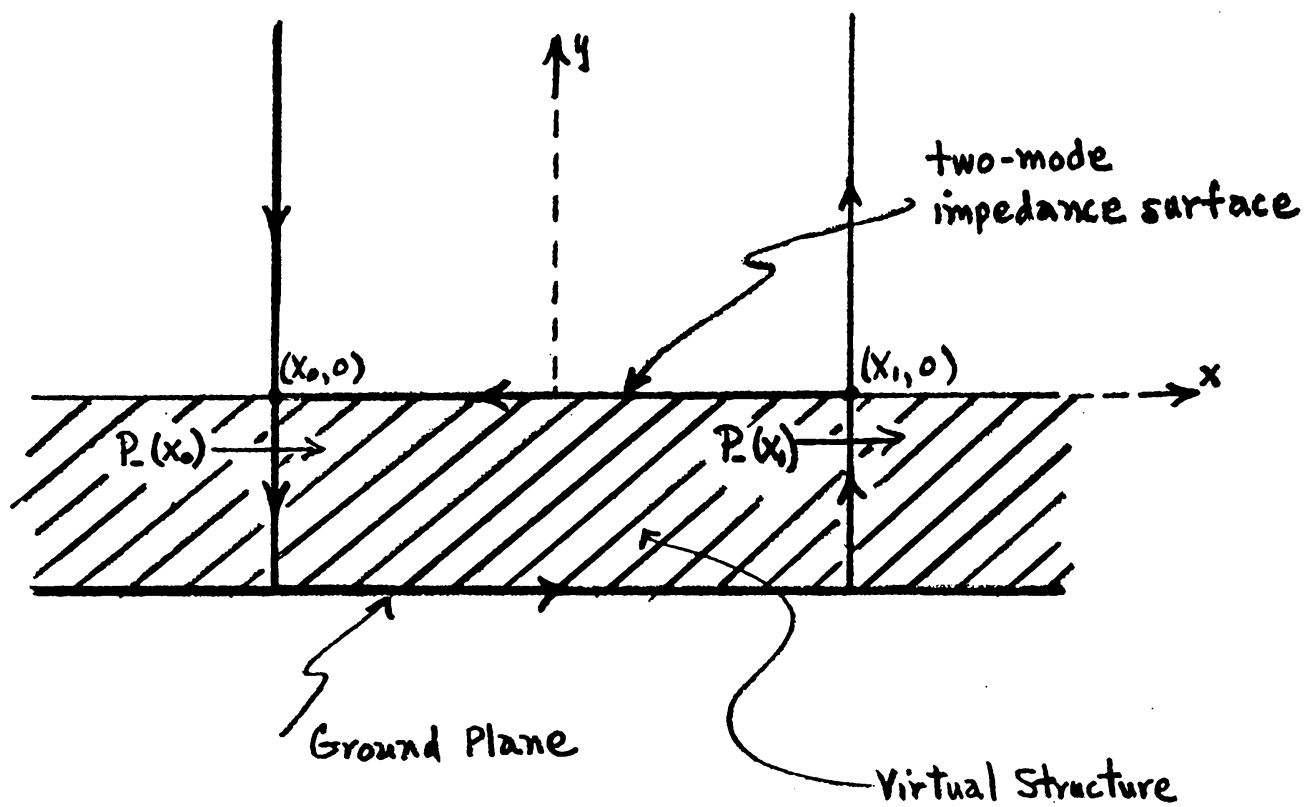

Fig. 4. The vertical contours used in the calculation of the surface wave power flow above the impedance structure are extended "beneath" the impedance surface at $\left(x_{0}, 0\right)$ and $\left(x_{1}, 0\right)$. Then conservation of power about the contour depicted by the arrows within this virtual structure is used to suggest a form of the power flow $(P-(x))$ through the extended portion of the contour.

the following difference equation:

$$
P_{-}\left(x_{0}\right)-\operatorname{Im} \frac{u\left(x_{0}, 0\right)}{\left(\lambda_{1}+\lambda_{2}\right)} \frac{\overline{\partial u}}{\partial x}\left(x_{0}, 0\right)=P_{-}\left(x_{1}\right)-\operatorname{Im} \frac{u\left(x_{1}, 0\right)}{\left(\lambda_{1}+\lambda_{2}\right)} \frac{\overline{\partial u}}{\partial x}\left(x_{1}, 0\right) .
$$

This has for its solution

$$
P_{-}(x)-\operatorname{Im} \frac{u(x, 0)}{\left(\lambda_{1}+\lambda_{2}\right)} \frac{\partial u}{\partial x}(x, 0)=+C,
$$

$C$ a constant. That is, the power flow across this extension of the vertical contour for any value of $x$ is given by

$$
P_{-}(x)=+\operatorname{Im} \frac{u(x, 0)}{\left(\lambda_{1}+\lambda_{2}\right)} \frac{\overline{\partial u}}{\partial x}(x, 0)+C .
$$

We observe that the first part of this expression, in addition to contributing to the power flow calculation, serves to remove the coupling term when the total power flow across the entire extended contour is considered to be

$$
\begin{aligned}
-\operatorname{Im} \int u \frac{\partial u}{\partial n} d s & =\operatorname{Im} \int_{+\infty}^{0} u \frac{\overline{\partial u}}{\partial x} d y+P_{-}(x) \\
& =\operatorname{Im}\left\{\int_{+\infty}^{0} u \frac{\partial u}{\partial x} d y+\frac{u(x, 0)}{\left(\lambda_{1}+\lambda_{2}\right)} \frac{\overline{\partial u}}{\partial x}(x, 0)\right\}+C .
\end{aligned}
$$

For convenience, we will let this extended power flow be denoted by $P_{B}[u ; x]$. (We will 
sometimes write this as $P_{E}(u)$, suppressing the $x$-dependence.) It will be shown that modal power flow separability will result only if we take $C$ to be zero.

Let us first observe that the boundary condition (ii) permits propagation of four different surface wave modes. For reference, we label them as follows:

$$
u_{i \pm} \equiv A_{ \pm}^{(i)} \exp \left[-\lambda_{i} y \pm i\left(k^{2}+\lambda_{i}^{2}\right)^{1 / 2} x\right], \quad j=1,2 .
$$

In addition, the power flow calculations will be simplified by defining the following form:

$$
(w, v)=\operatorname{Im}\left\{\int_{+\infty}^{0} w \frac{\overline{\partial v}}{\partial x} d y+\frac{w(x, 0)}{\left(\lambda_{1}+\lambda_{2}\right)} \frac{\overline{\partial v}}{\partial x}(x, 0)\right\}
$$

and observing that certain identities hold. Specifically; they are

$$
\begin{aligned}
& \left(u_{i \pm}, u_{l}\right)=0, \quad j_{ \pm} \neq l_{ \pm} \\
& \left(u_{i+}, u_{i-}\right)+\left(u_{i-}, u_{i+}\right)=0 \\
& \left(u_{i \pm}, u_{l_{ \pm}}\right)=\left|A_{ \pm}^{(i)}\right|^{2}\left(k^{2}+\lambda_{i}^{2}\right)^{1 / 2}\left(\frac{1}{2 \lambda_{i}}-\frac{1}{\lambda_{1}+\lambda_{2}}\right), \quad \lambda_{i \pm} \equiv \lambda_{l \pm}
\end{aligned}
$$

for $j=1,2$ and $l=1,2$. With these preliminaries, we will now compute the power flow of a field consisting only of modes of the type given in (3.18). Such a field is valid everywhere over a uniform two-mode structure. However, for the discontinuous problem of this paper, this field would hold only near the impedance plane at infinity. Thus, on setting

$$
u=u_{1+}+u_{1-}+u_{2+}+u_{2-}
$$

and applying (3.20), we find that

$$
P_{E}[u]=\left(u_{1+}, u_{1+}\right)+\left(u_{1-}, u_{1-}\right)+\left(u_{2+}, u_{2+}\right)+\left(u_{2-}, u_{2-}\right)+C .
$$

On the other hand, the modal power flow for each mode is given by

$$
P_{E}\left(u_{\text {mode }}\right)=\left(u_{\text {mode }}, u_{\text {mode }}\right)+C \text {. }
$$

We now apply modal power flow separability. By definition it holds if and only if

$$
P_{E}\left(u_{1+}+u_{1-}+u_{2+}+u_{2-}\right)=P_{E}\left(u_{1+}\right)+P_{E}\left(u_{1-}\right)+P_{E}\left(u_{2+}\right)+P_{E}\left(u_{2-}\right) .
$$

Thus, with (3.23) and (3.24) in mind, we are led to the conclusion that

$$
C=4 C
$$

or $C$ vanishes. Furthermore, as (3.16) holds for all $x<0$, we are now able to represent the power flow everywhere within the multiple-impedance section of the discontinuous structure by the formula

$$
P_{-}(x)=+\operatorname{Im} \frac{u(x, 0)}{\left(\lambda_{1}+\lambda_{2}\right)} \frac{\overline{\partial u}}{\partial x}(x, 0) .
$$

The reader is referred to [13] for another application of this point.

Allowing $R \rightarrow \infty$ in the contour integration results in the following modal surface 
wave power flows:

incident power flow:

$$
|A|^{2}\left(\frac{1}{2 \lambda_{1}}-\frac{1}{\lambda_{1}+\lambda_{2}}\right)\left(k^{2}+\lambda_{1}^{2}\right)^{1 / 2}
$$

excited power flow:

$$
-\sum_{m=1}^{2}\left|C_{m}\right|^{2}\left(\frac{1}{2 \lambda_{m}}-\frac{1}{\lambda_{1}+\lambda_{2}}\right)\left(k^{2}+\lambda_{m}^{2}\right)^{1 / 2}, \quad m=1,2
$$

where $\left|C_{m}\right|^{2}$ are given by (2.4) and (2.5). Note that the term involving $\lambda_{1}+\lambda_{2}$ represents a modification of the usual power flow formula for the incident and excited surface wave power terms. Also, we remark that it is easily observed that the total surface wave power is identical with (3.9). This latter fact will be exploited in the following section.

Power balance $C$. Conservation of power requires the integral given in (3.1) to be zero when evaluated around the closed contour of Fig. 2. As a partial check on our computations, this integral can be evaluated using the results previously stated for the surface wave and cylindrical radiated power under the special case of $R \rightarrow \infty, \theta(R) \rightarrow 0$, $R \sin \theta(R) \rightarrow \infty$, and $\rho \rightarrow 0$. Here the vertical integration contributes to the surface wave power and the integration over the circular contour $R=\left(x^{2}+y^{2}\right)^{1 / 2}$ becomes the radiated power. It was previously stated (in effect) that these two terms are absolutely equal but opposite in sign. On the other hand, the contribution due to $C_{\rho}$ is zero (as $\rho \rightarrow 0$ ) because of the boundedness of $u$ and $\partial u / \partial \rho$. Thus the only term that remains as a possible contributor is that of the boundary term $\lim _{\rho \rightarrow 0} \operatorname{Im}\left[\left(1 /\left(\lambda_{1}+\lambda_{2}\right)\right) u(-\rho, 0) \overline{(\partial u / \partial x)}(-\rho, 0)\right.$. However, it easily follows from the boundedness of $\partial u / \partial x$ and the continuity of $u$ that this term is identically zero. Thus, our results satisfy conservation of power. Furthermore, we can interpret these results to imply the following physical theorem: the incident surface wave power (including incident subsurface power flow) is equal to the excited surface wave power (including excited subsurface power flow) plus the cylindrical radiated power.

Conclusion. In this paper, we used a multiple impedance boundary condition to represent multi-mode surface wave diffraction by a discontinuous plane structure that is not amenable to an exact solution. Despite the complexity of our solution, which was obtained by a dual integral equation technique, we are able to determine the physically meaningful power flows as elementary functions of the impedance parameters.

A new feature of this paper is that it is shown how such multiple real impedance conditions may be used to model the power flow carried inside the structure itself. For problems modelled by the usual impedance condition, one never encounters this power flow because such problems usually represent zero power flow inside. However, it is well known that there are actual structures that exhibit substantial subsurface or internal power flows. In fact, the property of maintaining modal power flow separability for such exact structures requires that this power flow be taken into account. Interestingly, this same phenomenon was observed in this paper for the multiple impedance presented herein.

As a check on our various determinations of the power flow distributions, we verified that conservation of power holds around an appropriate contour. Additionally, this led us to state the following physical theorem for the magnitudes of the respective power 
flow distributions: incident surface wave power (including the associated subsurface power) equals the excited surface wave power (including the associated subsurface power) plus the cylindrically radiated power.

\section{REFERENCES}

[1] S. N. Karp and F. C. Karal, Phenonenological theory of muliti-mode surface wave structures, in Quasi-optics symposium, Brooklyn Polytechnic Institute, John Wiley, New York, 1964.

[2] F. C. Karal and S. N. Karp, Phenomenological theory of multi-mode surface waves for plane structures, Res. Rep. EM-198, Courant Institute of Mathematical Sciences, New York University, New York, 1964; condensed version, Quart. Appl. Math. 24, 239-247 (1966)

[3] R. C. Morgan, S. N. Karp, and F. C. Karal, Solution to the phenomenological problem of a magnetic line source above a plane structure that supports $N$ excited modes, SIAM J. Appl. Math. 15, 1363-1377 (1967)

[4] S. N. Karp and F. C. Karal, Generalized impedance boundary conditions with applications to surface wave structures, in Proc. LRSI, ('omm. VI Conference, Delft, The Netherlands, 1965.

[5] R. C. Morgan, S. N. Karp and F. C. Karal, Multi-mode surface wave diffraction by a right-angled wedge, Quart. Appl. Math. 24, 263-266 (1966)

[6] A. F. Kay, Scattering of a surface wave by a discontinuity in reactance, IEEE Trans. Antennas and Propagation AP-7, 22-31 (1959)

[7] J. Kane and S. N. Karp, Radio propagation past a pair of dielectric interfaces, Res. Rep. EM-154, Courant Institute of Mathematical Sciences, New York University, New York, 1960.

[8] J. Kane, Surface waves on a reactive half plane, Res. Rep. EM-159, Courant Institute of Mathematical Sciences, New York University, 1960.

[9] V. Fock, Sur certaines equations integrales de physique mathematique, Recueil Math. Nouvelle T.A. 56 (1944)

[10] J. Bazer and S. N. Karp, Propagation of plane electromagnetic waves past a shoreline, J. Res. Nat. Bur. Standards. 66D, 319-334 (1962)

[11] R. C. Morgan, Uniqueness theorem for a multi-mode surface wave diffraction problem, Quart. Appl. Math. 26, 601-604 (1969)

[12] R. E. Collin and F. J. Zucker, Antenna theory, pt. 2, McGraw-Hill, New York, 1969, p. 304.

[13] R. C. Morgan and S. N. Karp, Multi-mode surface wave phenomena, to appear. 